\title{
Neue Ansätze beim Management der Psoriasis- Arthritis: Können wir zielgerichtet behandeln?
}

\author{
Laura J. Tucker ${ }^{a}$ Weiyu Ye ${ }^{b}$ Laura C. Coates ${ }^{a}$ \\ ${ }^{a}$ Nuffield Department of Orthopaedics, Rheumatology and Musculoskeletal Sciences, Botnar Research Centre, University of Oxford, \\ Oxford, Großbritannien; ${ }^{\mathrm{b}}$ Oxford University Clinical Academic Graduate School, University of Oxford, Oxford, Großbritannien
}

\author{
Schlüsselwörter \\ Psoriasis-Arthritis · Treat-to-Target · Minimale Krankheitsaktivität . \\ Remission · Strenge Krankheitskontrolle · Biomarker
}

\begin{abstract}
Zusammenfassung
Zweck der Übersichtsarbeit: Psoriasis-Arthritis (PSA) ist eine chronisch entzündliche Spondyloarthritis, die zu fortschreitender Gelenkschädigung und irreversibler körperlicher Beeinträchtigung führen kann. Dank der Fortschritte der modernen Therapien ist die Remission heute ein erreichbares Behandlungsziel bei PsA. Es liegen belastbare, übereinstimmende Belege dafür vor, dass ein an konkreten Therapiezielen ausgerichteter Behandlungsansatz (Treat-to-Target; T2T) bei PsA zu besseren Outcomes für die Patienten führt. Die praktische Umsetzung dieses Ansatzes im klinischen Praxisalltag birgt allerdings noch einige Herausforderungen. Die Heterogenität des Krankheitsbildes und der Mangel an validierten Messgrößen haben bisher eine Konsensbildung über die Definition der Remission verhindert. Die vorliegende Arbeit soll einen Überblick über die aktuelle Forschungslandschaft im Bereich T2T bei
\end{abstract}

PsA geben und aufzeigen, welchen Beitrag Biomarker sowie eine moderne Radiologie zu einer grundlegenden Erneuerung des T2TKonzepts leisten können.

Aktuelle Erkenntnisse: Eine wachsende Evidenzbasis spricht für die Anwendung der T2T-Strategie, also der Ausrichtung der PsABehandlung an einem vorab festgelegten Ziel, und bescheinigt ihr signifikante Vorteile hinsichtlich des Krankheitsverlaufs, der körperlichen Funktionsfähigkeit und der Lebensqualität.

Fazit: Während die Remission unverändert das ultimative Ziel für PsA-Patienten und ihre behandelnden Ärzte bleibt, werden weitere Vergleichsstudien zu unterschiedlichen Therapiezielen benötigt, um eine allgemein akzeptable Definition der Remission zu finden.

(c) 2019 Die Autoren

\section{Einleitung}

Psoriasis-Arthritis (PsA) ist eine chronisch und progressiv verlaufende entzündliche Spondyloarthritis, von der bis zu 30\% aller Psoriasispatienten betroffen sind [1]. Die vielschichtige Krankheit kann eine große Bandbreite klinischer Erscheinungsformen annehmen, darunter Arthritis, Enthesitis, Daktylitis, Psoriasis und axiale Erkrankungen. Insoforn könnte man auch die Bezeichnung Psoriasis-Erkrankung als treffender erachten. Die PsA beeinträchtigt die Funktionsfähigkeit und Lebensqualität in ähnlichem Maße wie die rheumatoide Arthritis (RA) [2], und bei rund $20 \%$ der PsA-Patienten entwickeln sich irreversib- le Gelenkdeformitäten und bleibende Funktionseinschränkungen [3]. Es gibt zwar eine wachsende Evidenzbasis für die frühzeitige Erkennung und Behandlung der PsA [4-7], doch die Heterogenität des Krankheitsbildes erschwert die Umsetzung erheblich. Dank zunehmend besserer Kenntnis der Pathogenese konnten rasch wirksame, zielgerichtete Therapien entwickelt werden, die den Wirkungsbereich der PsA-Therapie grundlegend verändert und die Behandlungsmöglichkeiten erheblich vorangebracht haben. Das bedeutet nun, dass bei PsA ein Maß an Krankheitskontrolle zu einem realistischen Therapieziel geworden ist, das bisher als unerreichbar galt, wie z.B. eine Remission.

\section{KARGER}

(c) 2019 S. Karger GmbH, Freiburg

Fax +497614520714

information@karger.com

www.karger.com 
Das Konzept des Treat-to-Target (T2T) wurde ursprünglich für chronische Erkrankungen wie Diabetes mellitus, Hypertonie oder Hypercholesterinämie entwickelt und verbessert dort nachweislich die klinischen Behandlungsergebnisse [8-11]. Seit kurzem ist ein vergleichbarer Paradigmenwechsel auch auf dem Gebiet der Rheumatologie zu beobachten [12-14]; hier wurden durch Einführung des T2T-Ansatzes bei RA die Patienten-Outcomes regelrecht revolutioniert [12]. Die Ausweitung des Konzepts auch auf die PsA erfolgte mit der jüngst vorgestellten Studie TICOPA (TIght COntrol of Psoriatic Arthritis). Mit dieser Studie wurde erstmals der Nachweis erbracht, dass ein T2T-Vorgehen bei PsA bessere Behandlungsergebnisse erzielt als der Therapiestandard [15]. Angesichts der großen Heterogenität von PsA besteht jedoch Uneinigkeit darüber, welche Parameter des Ansprechens als Therapieziel gelten sollen, und so bleibt die praktische Umsetzung des $\mathrm{T} 2 \mathrm{~T}$ in der klinischen Routinepraxis auch weiterhin eine Herausforderung. Der vorliegende Bericht soll einen Überblick über die aktuelle Forschungslandschaft zum T2T-Konzept geben und das große Potenzial beschreiben, das Biomarker sowie die jüngsten Fortschritte in der Bildgebung im Hinblick auf die Anwendung bei PsA zeigen.

\section{Die Bedeutung des T2T-Konzeptes bei PsA}

T2T ist ein strategischer Ansatz, bei dem die Therapie auf ein vorab festgelegtes Ziel ausgerichtet ist. Mithilfe validierter Messgrößen wird regelmäßig und objektiv die Krankheitsaktivität beurteilt und die Therapie entsprechend angepasst, um das gesetzte Ziel zu erreichen. Dieser neuartige Ansatz entwickelt sich derzeit rasch zum Versorgungsstandard in der Rheumatologie. Bei RA etablierte sich das T2T-Konzept auf der Grundlage von Evidenz, die für einen Zusammenhang zwischen Entzündungsgeschehen und Gelenkschäden bei RA sprach [16]. Die TICORA-Studie zeigte dann, dass ein T2T-Vorgehen bei RA zu Verbesserungen bei der Krankheitsaktivität, dem radiologischen Fortschreiten, der körperlichen Funktionsfähigkeit und der Lebensqualität führte [12] Hiermit deckten sich auch die Ergebnisse einer Reihe anderer RAStudien (darunter die randomisierten kontrollierten Studien (RCT) CAMERA und STREAM), die so die Position des T2T im RA-Management festigten [17-23].

Inzwischen sind auch die Bereiche PsA und Spondyloarthritis (SpA) in den Fokus des Interesses gerückt. In den letzten 5 Jahren sind ausreichend Belege dafür gesammelt worden, dass die Entzündung eine Schlüsselrolle für die fortschreitende Gelenkschädigung bei PsA spielt $[24,25]$ und dass Schmerzen und Schwellung in einem Gelenk prädiktiv für den radiologischen Befund sind [26]. Das Ziel des T2T bei PsA ist es daher letztlich, die Gelenkentzündung zu eliminieren und struktureller Gelenkschädigung vorzubeugen, um so die gesundheitsbezogene Lebensqualität und die Funktionsfähigkeit zu maximieren und die körperliche Einschränkung zu minimieren. Und tatsächlich deuten Beobachtungsstudien darauf hin, dass eine frühe Diagnose und Therapieeinleitung bei PsA mit besseren klinischen, radiologischen und funktionellen Ergebnissen assoziiert sind [4-7].
Die TICOPA-Studie war die erste RCT, die den Nutzen einer T2TStrategie bei PsA bestätigte [15]. In der multizentrischen, unverblindeten RCT galt die an 7 Kriterien gemessene minimale Krankheitsaktivität (MDA) als das vorab festgelegte Ziel. MDA galt als gegeben, wenn 5 der folgenden 7 Kriterien gegeben waren: Anzahl schmerzender Gelenke $\leq 1$, Anzahl geschwollener Gelenke $\leq 1$, PASI (Psoriasis Area and Severity Index) $\leq 1$ oder BSA (Body Surface Area) $\leq 3$, Schmerzen laut Patientenbeurteilung auf einer visuellen Analogskala (VAS) $\leq 15$, globale Krankheitsaktivität laut Patientenbeurteilung $\leq 20$, HAQ-Score (Health Assessment Questionnaire) $\leq 0,5$ sowie Anzahl schmerzender enthesealer Punkte $\leq 1$ [27]. DMARD-naive erwachsene Patienten mit kürzlich aufgetretener PsA wurden per Randomisierung im Verhältnis 1:1 einer 48-wöchigen Behandlung entweder mit strenger Zielausrichtung oder nach einem standardmäßigen, nicht zielgerichteten Vorgehen zugeteilt (DMARD = disease-modifying antirheumatic drugs, Krankheitsverlauf modifizierende antirheumatische Medikamente). Die Patienten in der streng zielgerichteten Gruppe wurden alle 4 Wochen durch einen Studienrheumatologen untersucht, der die Therapie gemäß einem Algorithmus eskalierte, wenn keine MDA erreicht wurde. Die Patienten in der Standardtherapiegrupppe wurden alle 12 Wochen von ihrem jeweiligen behandelnden Rheumatologen untersucht und nach dessen Standard ohne einen Algorithmus behandelt. Die primäre Zielgröße der Studie war der Anteil der Patienten, bei denen in Woche 48 ein ACR20-Ansprechen (20\% Ansprechen laut American College of Rheumatology) vorlag. Die Eintretenswahrscheinlichkeit eines ACR20 war in der streng zielgerichtet behandelten Gruppe signifikant erhöht (Odds Ratio (OR) 1,91; $p=0,0392$ ); Gleiches galt für ACR50, ACR70 und PASI75. Auch die vom Patienten selbst beurteilten Parameter, wie z.B. die körperliche Funktionsfähigkeit und die Lebensqualität, verbesserten sich unter der strengen Zielausrichtung. In der Gruppe mit strenger Zielausrichtung waren höhere Raten von unerwünschten Ereignissen und schweren unerwünschten Ereignissen zu verzeichnen. Dies könnte auf die Anwendung von Kombinationstherapien und schneller Behandlungseskalation zurückzuführen sein oder es könnte zum Teil auch einen Reporting-Bias infolge häufigerer klinischer Untersuchungen widerspiegeln [15]. Bezüglich der längerfristigen Auswirkungen des T2T besteht noch Untersuchungsbedarf. Des Weiteren macht die erfolgreiche klinische Umsetzung der TICOPA-Studie eine Einigung über ein vorab festgelegtes, messbares Ziel für die PsA-Therapie erforderlich.

\section{Festlegung des Behandlungsziels}

Das heterogene Erscheinungsbild der PsA und der Mangel an validierten Verlaufsparametern erschweren die Konsensbildung über das optimale Therapieziel für den T2T-Ansatz bei PsA. Da biologische und zielgerichtete synthetische DMARD bei PsA hochgradig wirksam sein können, gibt es Stimmen, die sich für die Remission als optimales Therapieziel aussprechen [28-30]. Unter Remission wird die vollständige Abwesenheit jeglicher kutaner und sonstiger Krankheitsaktivität verstanden, begleitet von 
einem solchen Maß der Krankheitskontrolle, dass langfristige Schäden verhindert werden und die Lebensqualität erhalten bleibt [31]. Doch trotz zahlreicher Versuche, die Remission bei PsA zu definieren, wurde bisher keine allgemein akzeptierte Definition gefunden.

\section{Vergleich einiger zusammengesetzter Messgrößen zur Beurteilung der Krankheitsaktivität}

Die aus dem Bereich der RA abgeleiteten ACR-Ansprechkriterien und der DAS28 (28-Joint Disease Activity Score) sind nur sehr eingeschränkt geeignet, alle Krankheitsaspekte zu beurteilen [31, 32], und die Betrachtung einer geringeren Anzahl an Gelenken führt zur Fehleinschätzung von Patienten mit oligo- und monoartikulärer Erkrankung insbesondere der Sprung-, Fuß- und distalen Interphalangealgelenke [33]. Somit vernachlässigen diese Messgrößen einen erheblichen Anteil der Patienten mit aktiver psoriatischer Erkrankung. Einer kürzlich von der GRAPPAOMERACT-Gruppe durchgeführten Arztbefragung zufolge nutzen dennoch 19,5\% des medizinischen Fachpersonals den DAS28 weiterhin zur Feststellung einer Remission oder einer geringen Krankheitsaktivität (LDA; low disease activity) bei PsA [28].

2017 veröffentlichten Mease et al. [34] eine Analyse der vorliegenden Literatur zu Therapiezielen bei PsA (einschließlich MDA). Ihr Fazit lautete, dass der Facettenreichtum der PsA sich auch in den Behandlungszielen widerspiegeln und jede Zielsetzung die objektive Beurteilung von 5 Haupt-Krankheitsbereichen abdecken muss: Synovitis, Daktylitis, Enthesitis, Spondylitis und Psoriasis/ Nagelkrankheit [34]. Die beiden meistdiskutierten zusammengesetzten Messgrößen für PsA - die MDA und der DAPSA-Score (Disease Activity in PSoriatic Arthritis) - umfassen die Beurteilung aller 66/68 Gelenke. Beim DAPSA-Score wird jedoch nur die periphere Arthritis betrachtet, während die MDA-Kriterien relevante klinische Verlaufsparameter aus mehreren Bereichen abdecken. Diese Messgrößen finden in der klinischen und Forschungspraxis zunehmend Verbreitung [15, 25, 27, 35-37].

Die Festlegung von Cut-off-Werten dieser Scores als konkrete Behandlungsziele hat sich als praktikabel und akzeptabel erwiesen. So wurden folgende DAPSA-Werte bzw. -Wertebereiche als Kriterien für Remission und niedrige Krankheitsaktivität definiert und validiert: $\leq 4$ für Remission und $>4$ bis $\leq 14,>14$ bis $\leq 28$ und $>28$ für niedrige, mittlere bzw. hohe Krankheitsaktivität [35] Der DAPSA-Score lässt sich zwar schnell ermitteln, was die Eignung für den klinischen Alltag erhöht, doch er erfasst ausschließlich die Gelenkbeteiligung und bildet dadurch nur einen sehr engen Ausschnitt des psoriatischen Manifestationsspektrums ab. Der Parameter MDA hingegen umfasst die Betrachtung des gesamten Spektrums der psoriatischen Erkrankung und lässt sich mit einfachen Messgrößen für Enthesitis und Psoriasis zusätzlich zur peripheren Arthritis ebenfalls problemlos in die klinische Praxis einbinden. MDA liegt vor, wenn 5 der 7 Kriterien erfüllt sind. Sind alle 7 Kriterien erfüllt, spricht man von sehr geringer Krankheitsaktivität (VLDA; very low disease activity), die einer Remission entsprechen kann [27]. Der zusammengesetzte Parameter MDA ist jedoch lediglich eine binäre Größe, kann also den
Grad der Krankheitsaktivität bzw. des Ansprechens nicht auf einer kontinuierlichen Skala beziffern.

Das Therapieziel muss nicht nur in der klinischen Praxis mit vertretbarem Aufwand zu beurteilen sein, es muss auch erreichbar sein. Mehrere Studien belegen, dass bei den meisten PsA-Patienten, die mit einer Anti-Tumornekrosefaktor (TNF)-Therapie behandelt werden, der MDA-Status erreicht werden kann [38, 39]. Eine kürzlich in Spanien durchgeführte multizentrische Querschnittsstudie ergab, dass $60 \%$ der spanischen PsA-Patienten in der routinemäßigen klinischen Praxis MDA erreichten und die Auswirkung der Krankheit bei den Patienten mit MDA signifikant geringer waren [40]. Auch in einer kürzlich veröffentlichten norwegischen Studie wurden ähnliche Raten niedriger Krankheitsaktivität laut mehreren zusammengesetzten Messgrößen über einen Zeitraum von 10 Jahren ermittelt; bei den von den Patienten selbst beurteilten Parametern (PRO; patient-reported outcomes) waren jedoch keine signifikanten Verbesserungen in diesem Zeitraum zu verzeichnen. In dieser Studie wurde allerdings nicht zwischen den PRO-Ergebnissen der Patienten mit und denen ohne Remission verglichen [41].

Hierbei überrascht es wenig, dass verschiedene Studien zu zusammengesetzten Indizes ergeben haben, dass sich die geschätzten Remissionsraten je nach verwendeter zusammengesetzter Messgröße deutlich unterscheiden, und es liegen nur wenige Daten zu der Frage vor, welche Strategie angesichts der Risiken und Vorteile einer aggressiven zielgerichteten Therapie optimal ist [42]. Anhand der Datensätze von 250 PsA-Patienten, denen der jeweils behandelnde Rheumatologe eine ruhende Krankheit bescheinigt hatte, verglichen van Mens et al. [43] die zusammengesetzten Scores und stellten zwar einerseits signifikante Überschneidungen fest, andererseits aber auch Unterschiede im Hinblick auf die zulässige Residualerkrankung. Da beim DAPSA-Score die Erkrankung der Haut nicht betrachtet wird, gab es Fälle, die als «Remission» eingestuft waren, obwohl die Lebensqualität des Patienten durch residuelle Hauterkrankung beeinträchtigt war. In einer weiteren Untersuchung zeigten van Mens et al. [44] außerdem, dass Ärzte auch eine höhere Krankheitsaktivität als eine definitionsgemäße MDA akzeptabel fanden. Die Autoren stellten fest, dass ein Drittel der PsA-Patienten, deren Krankheitsstatus laut dem jeweils behandelnden Rheumatologen akzeptabel war, die MDA-Kriterien nicht erfüllten und dies mit erheblichen Beeinträchtigungen der Lebensqualität, Funktionsfähigkeit, psychischen Gesundheit und Arbeitsproduktivität einherging [44]. Diese Studie veranschaulicht die Diskrepanz zwischen der gegenwärtig üblichen klinischen Praxis und der zielwertbasierten Beurteilung und sollte alle Ärzte davon überzeugen, einfache Beurteilungsinstrumente wie die MDA oder den DAPSA-Score in ihre klinische Routine einzubinden.

\section{Krankheitsaktivität und Definition der Remission aus Patientensicht}

Es ist wichtig zu berücksichtigen, dass auch bei der Einschätzung der Krankheitsaktivität Diskrepanzen zwischen Patient und Arzt auftreten können. Für viele Patienten geht eine Remission des Ent- 
zündungsgeschehens nicht zwingend mit vollkommener Beschwerdefreiheit einher und spiegelt nicht die Beeinträchtigung der Lebensqualität und Funktionsfähigkeit durch die Resterkrankung wider. In einer Studie mit 565 PsA-Patienten untersuchten Eder et al. [45] die Diskrepanz zwischen den globalen Beurteilungen durch Patient und Arzt und stellten fest, dass die Patienten die Krankheit meist höher einstuften als die Ärzte. Ebenso ergab kürzlich die prospektive, multizentrische Studie NOR-DMARD Unterschiede im Patienten- und Arzturteil über die Krankheitsaktivität und die Anzahl schmerzender und geschwollener Gelenke, wobei bei den Patienten die Schmerzen stärker im Fokus standen. Die Autoren gelangten zu dem Schluss, dass diese Diskrepanz die Wahrscheinlichkeit einer Remission bei PsA verringern könnte [46].

Hierbei ist hervorzuheben, dass die Patienten eine Remission oft vor allem anhand der Auswirkungen der Krankheit auf ihre Lebensqualität und Funktionsfähigkeit definieren - Aspekte, die von vielen vom Patienten selbst beurteilten Messinstrumenten nicht immer ausreichend erfasst werden [47]. So stellten beispielsweise Tuyl et al. [48] in ihrer Studie zur RA fest, dass für die RAPatienten eine Remission eher ein Gefühl der Rückkehr zur Normalität war als eine Besserung der Symptome. Hier kann also eine Entkopplung in dem Sinne vorliegen, dass ein Patient seinen Krankheitsstatus nicht als Remission einstufen würde, jedoch nicht wegen der aktuellen Krankheitsaktivität, sondern wegen Gelenkschäden, Komorbiditäten oder chronischer Schmerzen.

Darüber hinaus sind die Erwartungen von einem Patienten zum nächsten ganz unterschiedlich und maßgeblich durch die konkrete Krankheitsmanifestation beeinflusst. Lubrano et al. [49] wiesen kürzlich nach, dass bei bis zu einem Viertel der Patienten, deren Krankheit mittels DAPSA und MDA als Remission oder LDA eingestuft wird, noch eine (meist kutane) Residualerkrankung vorliegt. Insofern ließe sich argumentieren, dass die sinnvollste Strategie nicht darin besteht, eine Remission oder LDA anzustreben, sondern darin, ein zu erreichendes Behandlungsziel zu definieren, das den für den individuellen Patienten wichtigsten Krankheitsaspekt abbildet.

\section{Aktuelle Empfehlungen zum T2T bei PsA}

Zu den Befürwortern der T2T-Strategie bei PsA zählen die internationale T2T-Taskforce, die European League Against Rheumatism (EULAR) und die Group for Research and Assessment of Psoriasis and Psoriatic Arthritis/Outcome Measures in Rheumatology (GRAPPA-OMERACT) [28-30, 50]. Die internationalen Empfehlungen unterstreichen die Unklarheiten bezüglich der Definition der Remission sowie der idealen zusammengesetzten Messgröße. Ärzte und Patienten sind sich jedoch darin einig, dass die Remission das primäre Behandlungsziel sein sollte bzw. unter bestimmten Umständen alternativ die LDA/MDA, z.B. bei seit langem bestehender Erkrankung, fixen Gelenkdeformitäten sowie bestimmten Komorbiditäten und Patientenzielen [28-30]. Solange keine eindeutigen Risiken gegen eine Eskalation der Therapie sprechen, sind höhere Stufen der Krankheitsaktivität nicht zu akzeptieren.
Eine aktuelle Patientenbefragung der GRAPPA-OMERACTGruppe ergab, dass 57\% des medizinischen Fachpersonals und 56\% der PsA-Patienten der Ansicht waren, dass die Remission das optimale Behandlungsziel sein sollte, mit LDA oder MDA als Alternative [28]. Außerdem fand die internationale T2T-Taskforce 2017 heraus, dass die große Mehrheit (88,9\%) der Befragten (Patientenvertreter, nichtmedizinisches Fachpersonal sowie rheumatologische und dermatologische Fachkräfte) der Empfehlung für die Remission/Inaktivität der Krankheit als primäres Behandlungsziel zustimmten [30]. Einige Befragte gaben an, dass progressive Gelenkschäden potenziell auch im Zustand der LDA auftreten können [24, 25], während der Begriff der Remission einen heilungsähnlichen Zustand ohne weitere Gelenkschäden bedeutete [30]. Entscheidend ist jedoch, dass nicht bekannt ist, ob die Remission als Therapieziel tatsächlich zu größeren Behandlungserfolgen bzw. besseren Patientenverläufen führt und welche potenziellen Risiken dies im Zuge einer aggressiveren Arzneimitteltherapie mit sich bringen kann.

\section{Umsetzung des T2T-Ansatzes in der klinischen Praxis}

Obwohl es PsA-spezifische Outcomes und Messgrößen gibt und internationale Empfehlungen zur standardmäßigen Anwendung der T2T-Strategie im PsA-Management aufrufen, lässt die weitreichende Umsetzung in die Praxis auf sich warten. Eine Arztbefragung der GRAPPA-OMERACT-Gruppe ergab, dass nur 56\% der Befragten in der klinischen Praxis bei PsA-Patienten den T2TAnsatz anwandten, wobei die MDA das am häufigsten verwendete Ziel war (32\%). Das zeigt, mit welcher Verzögerung Best Practices aus der Wissenschaft in die alltägliche klinische Praxis übernommen werden.

Innerhalb der Ärzteschaft besteht allgemeiner Konsens darüber, dass das Behandlungsziel individuell festzulegen ist, unter Berücksichtigung etwaiger Komorbiditäten sowie der Präferenzen der Patienten bezüglich der Krankheitsaktivität einerseits und der Sorgen und Bedenken der Patienten im Hinblick auf Arzneimittelsicherheit, kulturelle Einstellungen sowie arzneimittelassoziierte Risiken andererseits. Eine strenge Kontrolle der psoriatischen Erkrankung geht zwar mit vielfältigem Nutzen einher, ist aber auch nicht ohne Risiken. Die TICOPA-Studie hat gezeigt, dass die mit einer strengen Kontrolle verbundene intensivere Therapie auch mehr arzneimittelbedingte Nebenwirkungen hervorrufen kann [15]. Die Remission ist daher in bestimmten Situationen nicht immer das richtige Behandlungsziel, zum Beispiel bei Patienten, die stark zu wiederkehrenden Infektionen neigen. Zudem ist weithin anerkannt, dass eine strenge Remission bei vielen Patienten schwierig zu erreichen und aufrechtzuerhalten sein kann, insbesondere bei bereits lange bestehender Krankheit und bei Vorliegen von Folgeschäden und funktioneller Einschränkung oder von Komorbiditäten wie z.B. Adipositas [51]. Eine kürzlich durchgeführte Studie ergab, dass $42 \%$ aller PsA-Patienten $\geq 3$ Komorbiditäten haben [52]; dies ist somit eine beträchtliche potenzielle Hürde für die Erreichung des Remissionsziels. Unter diesen Umständen könnten daher auch MDA, «Beinahe- 
Remission» oder LDA als Ziel-Krankheitsstatus ausreichend sein [31].

Die Leitprinzipien der internationalen T2T-Taskforce besagen, dass Arzt und Patient gemeinsam über das Therapieziel entscheiden müssen, da die Remission nicht zwingend für alle Patienten das geeignete Ziel ist [30]. Eine Patientenbefragung der GRAPPAOMERACT-Gruppe ergab allerdings, dass die Mehrheit der PsAPatienten (61\%) ihre persönlichen Behandlungsziele nicht mit ihrem behandelnden Rheumatologen besprach und dass ein Fünftel der Patienten sich wünschte, dass der Rheumatologe sich mehr Zeit nehmen würde, ihnen zuzuhören [28]. Außerdem erwies sich die Patientenaufklärung bei PsA als verbesserungswürdig - PsAPatienten waren weniger aufgeklärt als RA-Patienten [53]. Letztlich ist die aktive Einbeziehung der Patienten in die Entscheidungsfindung über ihre künftige Behandlungsstrategie entscheidend für eine höhere Patientenzufriedenheit und die Umsetzung der T2T-Strategie im klinischen Alltag.

Um Patienten mit PsA optimal zu versorgen, müssen außerdem sämtliche Aspekte der psoriatischen Erkrankung berücksichtigt und mit validierten Instrumenten beurteilt werden, einschließlich des Ausmaßes und der Schwere der Hauterkrankung. Dies kann natürlich eine Herausforderung sein, wenn in der Klinik Zeitdruck herrscht. Doch während einige zusammengesetzte Messgrößen recht komplex und zeitaufwendig sind, sind sowohl der DAPSA-Score (zusammen mit validierten Messgrößen für Enthesitis und Psoriasis) als auch die MDA-Kriterien schnell zu erheben und auch in der Klinik einfach auszuwerten. Bei der Befragung der GRAPPA-OMERACT-Gruppe gab die Mehrheit der Ärzte an, die ideale zusammengesetzte Messgröße sollte ein guter Mittelweg zwischen der Testgüte und dem Zeitaufwand für die Erhebung sein und in weniger als 10 Minuten oder idealerweise in unter 5 Minuten durchführbar sein [28].

Die MDA-Kriterien sind derzeit die zusammengesetzte Messgröße, die psoriatische Erkrankungen am besten abbildet und gleichzeitig in der klinischen Praxis praktikabel ist. Es handelt sich um einen Patientenfragebogen, den die Patienten im Wartezimmer vor einem Arzttermin ausfüllen können; hierfür benötigen sie etwa 5-10 Minuten. Derzeit werden weniger als 25\% der Patienten gebeten, vor ihren Arztterminen Fragebögen auszufüllen, während $91 \%$ bereit wären, dies zu tun, wenn sie darum gebeten würden [28]. Die MDA-Erhebung ist eine relativ kurze Evaluierung; bei regelmäßiger Anwendung durch den Kliniker kann der Zeitbedarf für die klinische Beurteilung verschiedener Krankheitsaspekte 5-10 Minuten betragen [54].

Um eine zeitgemäße Anpassung der Behandlung zu ermöglichen, darf die Beurteilung der Krankheitsaktivität natürlich kein singuläres Ereignis bleiben, sondern muss regelmäßig erfolgen. Es mangelt jedoch derzeit noch an Daten dazu, welches Zeitintervall für das Monitoring das beste ist. Die Patienten im TICOPA-Studienarm mit strenger Zielausrichtung wurden im Abstand von 4 Wochen untersucht [15]. In der klinischen Praxis ist dies bei Patienten, deren Therapie erst kürzlich umgestellt wurde oder die ihr vorab festgelegtes Behandlungsziel erreicht haben, weder praktikabel noch notwendig. Die EULAR-Empfehlungen für das
PsA-Management von 2015 sehen eine «regelmäßige Überwachung» von Patienten mit PsA vor und empfehlen bei aktiver Erkrankung ein Monitoring im Abstand von 1-3 Monaten [29]. Gladman et al. [55] legten kürzlich Empfehlungen zur Anwendung von T2T in der klinischen Praxis in Kanada vor; sie empfehlen, dass bei Patienten mit aktiver Krankheit alle 3 Monate eine Untersuchung in der Klinik sowie eine Anpassung der Medikation erfolgen sollte und bei Patienten, die ihre therapeutischen Ziele erreicht haben, alle 6-12 Monate. Hierbei bleibt noch zu untersuchen, ob die geringere Häufigkeit der Besuche beim Rheumatologen die positiven Effekte des T2T verwässert.

\section{Kosteneffizienz von T2T-Strategien}

Die Schwere und die Chronizität der PsA schlagen sich auch in hohen direkten und indirekten Gesundheitskosten nieder [56, 57]. So liegen die Kosten in Großbritannien zwischen 11 und 20782 GBP pro Patient und Jahr; die durchschnittlichen jährlichen Gesundheitskosten pro Biologika-naivem PsA-Patienten in Großbritannien betragen 1446 GBP [58]. Diese Kostenangaben decken sich mit Studien zur Beurteilung der finanziellen Auswirkungen der PsA in anderen europäischen Ländern [56, 59]; in den USA sind die direkten Gesundheitskosten der PsA höher. In einer US-Studie mit Daten aus den Jahren 2011 und 2012 wurden die durchschnittlichen direkten Kosten auf 19282 USD pro Patient und Jahrgeschätzt; bereinigtumbiologischeDMARD(bDMARD) entspricht dies jährlichen Kosten von 5258 USD pro Patient [60]. Steigende allgemeine Gesundheitskosten bei fest begrenzten staatlichen Budgets erzeugen einen zunehmend hohen Druck, eine klinisch effektive Gesundheitsversorgung zu geringstmöglichen Kosten zu leisten. Der T2T-Ansatz ist bei RA bereits besser etabliert und hat insbesondere in den frühen Stadien der RA viele Fürsprecher, bei längerem Krankheitsverlauf hingegen erheblich weniger, weshalb hier tragfähigere Evidenz zur Wirksamkeit und Kosteneffizienz von T2T-Strategien gefordert wird.

Während eine strenge Zielausrichtung der Therapie sich in der TICOPA-Studie als klinisch wirksam erwies [15], ergaben die Kosten-Nutzen-Analysen der strengen Zielausrichtung im Vergleich zur Standardversorgung, dass die strenge Zielausrichtung in dieser Studie nicht kosteneffizient war [15]. Es wurde nur ein geringfügiger Unterschied bei den QALY (um die Lebensqualität adjustierten Lebensjahren) festgestellt, die durch strenge Zielausrichtung im Vergleich zur Standardtherapie gewonnen wurden [61], allerdings wurde dies nur über 48 Wochen gemessen. Eine Sensitivitätsanalyse unter Berücksichtigung sinkender Arzneimittelkosten durch vermehrte Anwendung von Biosimilars sowie eine geringere Häufigkeit von Rheumatologenbesuchen nach Etablierung der Erhaltungstherapie deuteten auf eine verbesserte Kosteneffizienz im akzeptablen Bereich hin. Da zudem Polyarthritis-Patienten von einer T2T-Behandlung stärker profitierten [15, 61], lässt sich die Kosteneffizienz dieses Ansatzes möglicherweise durch eine noch zielgenauere Anwendung optimieren. Unter dem Strich sind längerfristige Nachbeobachtungsstudien und Daten aus der praktischen Anwendung des T2T-Ansatzes in 
der klinischen Praxis unverzichtbar, um die Kosteneffizienz zu beurteilen und zu prüfen, ob der zusätzliche klinische Nutzen einer strengen Zielausrichtung Bestand hat und sich in einem $\mathrm{Zu}$ gewinn an QALY niederschlägt.

Gibt es einen Platz für immunologische und radiologische Marker in der Definition der Remission?

\section{Biomarker der Krankheitsaktivität}

Trotz signifikanter Fortschritte bei den «omischen» Molekulartechnologien in den letzten 10 Jahren gibt es bisher keine validierten Biomarker, um psoriatische Erkrankungen zu diagnostizieren, das Ansprechen auf Therapien vorherzusagen oder Patienten in Remission zu identifizieren. Zwar werden Standard-Akutphasen-Reaktanden wie das C-reaktive Protein (CRP) oder die Blutsenkungsgeschwindigkeit (BSG) als Marker für die Krankheitsaktivität bei PsA genutzt, jedoch sind sie nicht spezifisch für psoriatische Erkrankungen. Überzeugende Evidenz spricht dafür, dass der Interleukin (IL)-17/IL-23-Signalweg in der Pathogenese sowohl der Psoriasis als auch der PsA eine zentrale Rolle spielt [62-68]. Menon et al. [63] wiesen nach, dass Synovialflüssigkeit von PsA-Patienten einen erhöhten Anteil von Th17 und IL-17 CD8+ T-Zellen (Tc17) enthält, sowohl im Vergleich zum peripheren Blut als auch im Vergleich zu Synovialflüssigkeit von RA-Patienten, bei denen die Th17-, nicht aber die Tc17-Zellen im Vergleich zum peripheren Blut erhöht sind. Darüber hinaus fanden sie heraus, dass der Anteil der Tc17-Zellen in der Synovialflüssigkeit von PsA-Patienten positiv mit Markern für Krankheitsaktivität sowie dem radiologischen Erosionsstatus nach 2 Jahren korrelierte [63].

Eine Reihe von frei im Serum vorliegenden Markern für Knochen- und Knorpelumsatz bei PsA und psoriatischer Spondyloarthritis wurden als potenzielle Krankheitsbiomarker identifiziert. Hierzu zählen Matrixmetalloproteinase 3 (MMP-3), Dickkopf 1 (DKK-1), Makrophagenkolonien stimulierende Faktoren (M-CSF) und Osteoprotegerin (OPG) [69, 70]. Eine aktuelle prospektive Querschnittsvergleichsstudie hat bestätigt, dass diese 4 Biomarker mit PsA assoziiert sind. MMP-3 und M-CSF erwiesen sich als Biomarker für das Vorliegen von Psoriasis bei psoriatischen Erkrankungen; sie könnten zum Screening auf PsA bei Psoriasispatienten nutzbar sein. Die DKK-1- und OPG-Konzentrationen könnten helfen, zwischen PsA-Patienten mit und ohne axiale Arthritis zu unterscheiden, und somit im Screening von PsA-Patienten auf axiale Erkrankung eingesetzt werden; darüber hinaus könnten sie zur Unterscheidung zwischen psoriatischer Spondyloarthritis und ankylosierender Spondylitis beitragen [71]. Longitudinalstudien zur Validierung dieser Biomarker der Krankheitsaktivität sind erforderlich.

Fortschritte in der Erforschung der Pathobiologie der PsA werden zur Identifizierung neuer Biomarker für psoriatische Erkrankungen ebenso beitragen wie zur Definition der immunologischen Remissionen im Rahmen des T2T, und sie werden es den Ärzten ermöglichen, wirksamer und individueller zu behandeln.
Einsatz der Bildgebung zur Überwachung der Krankheitsaktivität und Definition der Remission

Moderne bildgebende Verfahren wie Ultraschall (US) und Magnetresonanztomografie (MRT) haben unser Verständnis der Pathogenese der verschiedenen PsA-Phänotypen erweitert und sinnvolle Beiträge zur Diagnostik psoriatischer Erkrankungen schon im subklinischen Rahmen geleistet [72]. Beide Bildgebungsmodalitäten erlauben eine frühzeitige Erkennung entzündlicher Veränderungen in Gelenken und benachbarten Strukturen sowie die Beurteilung des Ausmaßes struktureller Schädigung [72-77]. Bei der Identifizierung bestimmter PsA-Pathologien zeigen sie höhere Sensitivität als die klinische Untersuchung [75]. Im US erkennbare Synovitis und Enthesitis sind mit langfristiger radiologischer Progression und schlechtem Verlauf assoziiert [78, 79]. Während diese bildgebenden Verfahren bei Psoriasis bisher vor allem für die Diagnostik genutzt wurden, kommen sie sowohl in der klinischen als auch in der Forschungspraxis zunehmend auch zur objektiven Messung des Ansprechens auf die Behandlung zum Einsatz. Und auch die EULAR empfiehlt diese Praxis [80].

Diese hochgradig sensitiven Bildgebungsverfahren haben das Potenzial, das PsA-Management zu verbessern; doch ihre genaue Rolle bei der Definition der Remission bei PsA sowie der Abbildung subklinischer Krankheitsaktivität ist noch nicht geklärt. Für die RA wurde dies bereits umfassender untersucht; hier verglich die ARCTIC-Studie 2 Behandlungsstrategien mit strenger Zielausrichtung bei früher RA, um zu ermitteln, ob eine auf einer strukturierten US-Evaluierung basierende Behandlungsstrategie bei RA zu besseren Outcomes führen würde. 122 Patienten wurden per Randomisierung einem Vorgehen mit US und strenger Zielausrichtung auf klinische und radiologische Remission zugeteilt, weitere 116 Patienten erhielten eine konventionelle Therapie mit strenger Ausrichtung auf das Ziel der klinischen Remission. Trotz aggressiverer Behandlung in der US-Gruppe waren zwischen beiden Gruppen keine Unterschiede im Hinblick auf Gelenkschwellung, klinische Remission oder Inhibition radiologischer Gelenkschädigung zu verzeichnen [81]. Ebenso ergab die TaSER-Studie, dass eine US-basierte T2T-Strategie in einer intensiveren Therapie resultierte, jedoch nicht in signifikant besseren klinischen oder radiologischen Verläufen [82]. Unter dem Strich gelangten beide Studien zu dem Schluss, dass der Einsatz von US in der routinemäßigen Verlaufsbeobachtung von Patienten mit RA in frühen Stadien nicht gerechtfertigt sei, und die ARCTICStudie lieferte zudem Hinweise darauf, dass durch den mangelnden Nutzengewinn und den höheren Kosten- und Zeitaufwand sowie die vermehrte Anwendung von bDMARD, die mit dem USbasierten Therapieschema einhergehen, die Kosten-Nutzen-Relation negativ ausfallen würde [81]. Diese Studienergebnisse überraschen ein wenig angesichts der wachsenden Evidenzbasis dafür, dass eine subklinische Entzündung mit radiologischer Progression und Krankheitsflares assoziiert ist [78, 79, 83-85]. Weitere Studien müssen die Korrelation zwischen sonografischer und histologisch bestätigter Entzündung untersuchen und die Wirksamkeit des US im PsA-Management beurteilen. Derzeit gibt es keine 
ausreichende Evidenz dafür, die US-Untersuchung routinemäßig in die T2T-Strategie bei neu diagnostizierter entzündlicher Arthritis aufzunehmen.

\section{Schlussfolgerungen}

Eine wachsende Evidenzbasis spricht für das T2T-Konzept bei PsA. Mit den Fortschritten der modernen Therapie ist es heute ein realistisches Ziel, einen Krankheitsstatus zu erreichen, bei dem Progression und Gelenkschäden vermieden werden und die langfristige Funktionsfähigkeit optimiert wird. Es muss jedoch noch eine Definition der Remission gefunden werden, die für Patienten, Kliniker und Forscher gleichermaßen akzeptabel ist. Letztlich wird die gemeinsame Entscheidungsfindung durch Patienten und Rheumatologen entscheidend dafür sein, die Patientenzufriedenheit und das Management dieser chronischen Erkrankung zu verbessern.

Fortschritte bei den «omischen» Molekulartechnologien werden ein besseres Verständnis der Signalwege ermöglichen, die an der Pathogenese der PsA beteiligt sind, und somit der Schlüssel zur Entdeckung spezifischer neuer Biomarker und zur Definition der immunologischen Remission bei psoriatischen Erkrankungen sein. Moderne bildgebende Verfahren haben sich als Bereicherung in der Diagnostik der Psoriasis bewährt - ob die radiologische Remission auch einen zusätzlichen Nutzen zu zusammengesetzten klinischen Messgrößen besitzt, bleibt noch zu klären.
T2T ist ein spannendes und rapide voranschreitendes Forschungsgebiet. In künftigen Vergleichsstudien muss untersucht werden, wie erfolgreich neue Therapieziele genutzt werden können, ob die Einhaltung strengerer Ziele wie z.B. Remission den klinischen Nutzen vergrößert und ob langfristige Verbesserungen sich auch in der Kosteneffizienz niederschlagen.

\section{Finanzierung}

L.J.T. erhält Mittel von der Norman Collisson Foundation als Clinical Research Fellow. W.Y. ist Trainee einer akademischen Stiftung. L.C.C. erhält Mittel durch einen National Institute for Health Research Clinician Scientist Award. Diese Arbeit wurde durch das National Institute for Health Research (NIHR) Oxford Biomedical Research Centre (BRC) unterstützt. Die hier geäußerten Ansichten der Autoren stimmen nicht unbedingt mit denen der National Health Services (NHS), des NIHR oder des Gesundheitsministeriums überein.

\section{Disclosure Statement}

Die Autoren erklären, dass keine Interessenkonflikte bestehen.

\section{Lizenzangabe}

Laura J. Tucker, Weiyu Ye, Laura C. Coates: Novel concepts in psoriatic arthritis management: Can we treat to target? Curr Rheumatol Rep 2018; 20:71 (https://doi.10.1007/s11926-018-0781-x). @ The Author(s) 2018 (Übersetzung, «Human and Animal Rights and Informed Consent» gekürzt), lizensiert unter CC-BY 4.0 (https://creativecommons.org/licenses/by/4.0/ deed.de).

\section{Literatur}

1 Mease PJ, Gladman DD, Papp KA, Khraishi MM, Thaçi D, Behrens F, Northington R, Fuiman J, Bananis E, Boggs R, Alvarez D: Prevalence of rheumatologist-diagnosed psoriatic arthritis in patients with psoriasis in European/North American dermatology clinics. J Am Acad Dermatol 2013;69:729-735.

-2 Sokoll KB, Helliwell PS: Comparison of disability and quality of life in rheumatoid and psoriatic arthritis. J Rheumatol 2001;28:1842-1846.

$\checkmark 3$ Kane D et al.: A prospective, clinical and radiological study of early psoriatic arthritis: an early synovitis clinic experience. Rheumatology (Oxford) 2003;42:1460-1468.

4 Theander E, Husmark T, Alenius G-M, Larsson PT, Teleman A, Geijer M, Lindqvist UR: Early psoriatic arthritis: short symptom duration, male gender and preserved physical functioning at presentation predict favourable outcome at 5-year follow-up. Results from the Swedish Early Psoriatic Arthritis Register (SwePsA). Ann Rheum Dis 2013;73:407-413.

$\checkmark 5$ Tillett W, Jadon D, Shaddick G, Cavill C, Korendowych E, de Vries CS, McHugh N: Smoking and delay to diagnosis are associated with poorer functional outcome in psoriatic arthritis. Ann Rheum Dis 2013;72:1358-1361.

6 Gladman DD, Thavaneswaran A, Chandran V, Cook RJ: Do patients with psoriatic arthritis who present early fare better than those presenting later in the disease? Ann Rheum Dis 2011;70:2152-2154.
7 Haroon M, Gallagher P, FitzGerald O: Diagnostic delay of more than 6 months contributes to poor radiographic and functional outcome in psoriatic arthritis. Ann Rheum Dis 2015;74:1045-1050.

$\checkmark$ Hansson L, Zanchetti A, Carruthers SG, Dahlöf B, Elmfeldt D, Julius S, Ménard J, Rahn $\mathrm{KH}$, Wedel H, Westerling S: Effects of intensive blood-pressure lowering and low-dose aspirin in patients with hypertension: principal results of the Hypertension Optimal Treatment (HOT) randomised trial. Lancet 1998;351: 1755-1762.

9 Nathan DM et al.: The effect of intensive treatment of diabetes on the development and progression of long-term complications in insulin-dependent diabetes mellitus. N Engl J Med 1993;329:977-986.

10 Warram JH, Manson JE, Krolewski AS: Glycosylated hemoglobin and the risk of retinopathy in insulin-dependent diabetes mellitus. N Engl J Med 1995;332:1305-1306.

11 Ridker PM: Moving toward new statin guidelines in a post-JUPITER world: principles to consider. Curr Atheroscler Rep 2009;11: 249-256.

12 Grigor C, Capell H, Stirling A, McMahon AD, Lock P, Vallance R, Porter D, Kincaid W: Effect of a treatment strategy of tight control for rheumatoid arthritis (the TICORA study): a single-blind randomised controlled trial. Lancet 2004;364:263-269.
3 van Vollenhoven RF, Mosca M, Bertsias G, Isenberg D, Kuhn A, Lerstrøm K, et al.: Treat-totarget in systemic lupus erythematosus: recommendations from an international task force. Ann Rheum Dis 2014;73:958-967.

14 Kiltz U, Smolen J, Bardin T, Cohen Solal A, Dalbeth N, Doherty M, Engel B, Flader C, Kay J, Matsuoka M, Perez-Ruiz F, da Rocha CastelarPinheiro G, Saag K, So A, Vazquez Mellado J, Weisman M, Westhoff TH, Yamanaka H, Braun J: Treat-to-target (T2T) recommendations for gout. Ann Rheum Dis 2016;76:632-638.

15 Coates LC et al.: Effect of tight control of inflammation in early psoriatic arthritis (TICOPA): a UK multicentre, open-label, randomised controlled trial. Lancet 2015;386: 2489-2498.

16 Stenger AA et al.: Early effective suppression of inflammation in rheumatoid arthritis reduces radiographic progression. Br J Rheumatol 1998;37:1157-1163.

17 Verstappen SM et al.: Intensive treatment with methotrexate in early rheumatoid arthritis: aiming for remission. Computer Assisted Management in Early Rheumatoid Arthritis (CAMERA, an open-label strategy trial). Ann Rheum Dis 2007;66:1443-1449.

18 Fransen J et al.: Effectiveness of systematic monitoring of rheumatoid arthritis disease activity in daily practice: a multicentre, cluster randomised controlled trial. Ann Rheum Dis 2005;64:1294-1298. 
19 Vermeer M, Kievit W, Kuper HH, BraakmanJansen LMA, Bernelot Moens HJ, Zijlstra TR, et al.: Treating to the target of remission in early rheumatoid arthritis is cost-effective: results of the DREAM registry. BMC Musculoskelet Disord 2013;14:350.

-20 Schipper LG, Vermeer M, Kuper HH, Hoekstra MO, Haagsma CJ, Den Broeder AA, van Riel P, Fransen J, van de Laar MA: A tight control treatment strategy aiming for remission in early rheumatoid arthritis is more effective than usual care treatment in daily clinical practice: a study of two cohorts in the Dutch Rheumatoid Arthritis Monitoring registry. Ann Rheum Dis 2011;71:845-850.

21 van Eijk IC, Nielen MMJ, van der Horst-Bruinsma I, Tijhuis GJ, Boers M, Dijkmans BAC, et al.: Aggressive therapy in patients with early arthritis results in similar outcome compared with conventional care: the STREAM randomized trial. Rheumatology (Oxford) 2012;51: 686-694.

-22 Soubrier M, Lukas C, Sibilia J, Fautrel B, Roux F, Gossec L, Patternotte S, Dougados M: Disease activity score-driven therapy versus routine care in patients with recent-onset active rheumatoid arthritis: data from the GUEPARD trial and ESPOIR cohort. Ann Rheum Dis 2011;70:611-615.

-23 Goekoop-Ruiterman YP, de Vries-Bouwstra JK, Kerstens PJ, Nielen MM, Vos K, van Schaardenburg D, Speyer I, Seys PE, Breedveld FC, Allaart CF, Dijkmans BA: DAS-driven therapy versus routine care in patients with recent-onset active rheumatoid arthritis. Ann Rheum Dis 2009;69:65-69.

-24 Cresswell L, Chandran V, Farewell VT, Gladman DD: Inflammation in an individual joint predicts damage to that joint in psoriatic arthritis. Ann Rheum Dis 2011;70:305-308.

$\checkmark 25$ Aletaha D, Alasti F, Smolen JS: Disease activity states of the DAPSA, a psoriatic arthritis specific instrument, are valid against functional status and structural progression. Ann Rheum Dis 2017;76:418-421.

26 Bond SJ, Farewell VT, Schentag CT, Gladman DD: Predictors for radiological damage in psoriatic arthritis: results from a single centre. Ann Rheum Dis 2007;66:370-376.

27 Coates LC, Fransen J, Helliwell PS: Defining minimal disease activity in psoriatic arthritis: a proposed objective target for treatment. Ann Rheum Dis 2010;69:48-53.

28 Coates LC, FitzGerald O, Merola JF, Smolen J, van Mens LJJ, Bertheussen H, Boehncke W-H, Callis Duffin K, Campbell W, de Wit M, Gladman D, Gottlieb A, James J, Kavanaugh A, Kristensen LE, Kvien TK, Luger T, McHugh N, Mease P, Nash P, Ogdie A, Rosen CF, Strand V, Tillett W, Veale DJ, Helliwell PS: Group for Research and Assessment of Psoriasis and Psoriatic Arthritis/Outcome Measures in Rheumatology consensus-based recommendations and research agenda for use of composite measures and treatment targets in psoriatic arthritis. Arthritis Rheumatol 2018;70:345-355.

-29 Gossec L et al.: European League Against Rheumatism (EULAR) recommendations for the management of psoriatic arthritis with pharmacological therapies: 2015 update. Ann Rheum Dis 2016;75:499-510.
30 Smolen JS et al.: Treating axial spondyloarthritis and peripheral spondyloarthritis, especially psoriatic arthritis, to target: 2017 update of recommendations by an international task force. Ann Rheum Dis 2018;77:3-17.

31 Kavanaugh A, Fransen J: Defining remission in psoriatic arthritis. Clin Exp Rheumatol 2006;24(6 suppl 43):S-83-S-87.

32 Caperon A, Helliwell PS: Remission in psoriatic arthritis. J Rheumatol Suppl 2012;89: 19-21.

33 Coates LC, FitzGerald O, Gladman DD, McHugh N, Mease P, Strand V, et al.: Reduced joint counts misclassify patients with oligoarticular psoriatic arthritis and miss significant numbers of patients with active disease. Arthritis Rheum 2013;65:1504-1509.

34 Mease PJ, Coates LC: Considerations for the definition of remission criteria in psoriatic arthritis. Semin Arthritis Rheum 2018;47: 786-796.

-35 Schoels M, Aletaha D, Funovits J, Kavanaugh A, Baker D, Smolen JS: Application of the DAREA/DAPSA score for assessment of disease activity in psoriatic arthritis. Ann Rheum Dis 2010;69:1441-1447.

36 Coates LC, Helliwell PS: Validation of minimal disease activity criteria for psoriatic arthritis using interventional trial data. Arthritis Care Res (Hoboken) 2010;62:965-969.

37 Schoels MM, Aletaha D, Alasti F, Smolen JS: Disease activity in psoriatic arthritis (PsA): defining remission and treatment success using the DAPSA score. Ann Rheum Dis 2016;75: 811-818.

38 Haddad A, Thavaneswaran A, Ruiz-Arruza I, Pellett F, Chandran V, Cook RJ, Gladman DD: Minimal disease activity and anti-tumor necrosis factor therapy in psoriatic arthritis. Arthritis Care Res 2015;67:842-847.

39 Mease PJ, Heckaman M, Kary S, Kupper H: Application and modifications of minimal disease activity measures for patients with psoriatic arthritis treated with adalimumab: subanalyses of ADEPT. J Rheumatol 2013;40: 647-652.

40 Queiro R et al.: Minimal disease activity and impact of disease in psoriatic arthritis: a Spanish cross-sectional multicenter study. Arthritis Res Ther 2017;19:72.

41 Haugeberg G, Michelsen B, Tengesdal S, Hansen IJW, Diamantopoulos A, Kavanaugh A: Ten years of follow-up data in psoriatic arthritis: results based on standardized monitoring of patients in an ordinary outpatient clinic in southern Norway. Arthritis Res Ther 2018;20: 160.

42 Salaffi $\mathrm{F}$ et al.: Disease activity in psoriatic arthritis: comparison of the discriminative capacity and construct validity of six composite indices in a real world. Biomed Res Int 2014; 2014:528105.

43 van Mens LJJ, van de Sande MGH, van Kuijk AWR, Baeten D, Coates LC: Ideal target for psoriatic arthritis? Comparison of remission and low disease activity states in a real-life cohort. Ann Rheum Dis 2018;77:251-257.
44 van Mens LJJ, Turina MC, van de Sande MGH, Nurmohamed MT, van Kuijk AWR, Baeten DLP: Residual disease activity in psoriatic arthritis: discordance between the rheumatologist's opinion and minimal disease activity measurement. Rheumatology (Oxford) 2018; 57:283-290.

45 Eder L, Thavaneswaran A, Chandran V, Cook R, Gladman DD: Factors explaining the discrepancy between physician and patient global assessment of joint and skin disease activity in psoriatic arthritis patients. Arthritis Care Res (Hoboken) 2015;67:264-272.

46 Michelsen B et al.: Discordance between tender and swollen joint count as well as patient's and evaluator's global assessment may reduce likelihood of remission in patients with rheumatoid arthritis and psoriatic arthritis: data from the prospective multicentre NOR-DMARD study. Ann Rheum Dis 2017;76:708-711.

47 Stamm TA, Nell V, Mathis M, Coenen M, Aletaha D, Cieza A, Stucki G, Taylor W, Smolen JS, Machold KP: Concepts important to patients with psoriatic arthritis are not adequately covered by standard measures of functioning. Arthritis Rheum 2007;57:487-494.

48 van Tuyl LH et al.: The patient perspective on remission in rheumatoid arthritis: 'You've got limits, but you're back to being you again'. Ann Rheum Dis 2015;74:1004-1010.

49 Lubrano E, De Socio A, Perrotta FM: Comparison of composite indices tailored for psoriatic arthritis treated with csDMARD and bDMARD: a cross-sectional analysis of a longitudinal cohort. J Rheumatol 2017;44:1159-1164.

50 van der Heijde D et al.: 2016 update of the ASAS-EULAR management recommendations for axial spondyloarthritis. Ann Rheum Dis 2017;76:978-991.

51 Eder L, Thavaneswaran A, Chandran V, Cook RJ, Gladman DD: Obesity is associated with a lower probability of achieving sustained minimal disease activity state among patients with psoriatic arthritis. Ann Rheum Dis 2015;74: 813-817.

52 Husted JA, Thavaneswaran A, Chandran V, Gladman DD: Incremental effects of comorbidity on quality of life in patients with psoriatic arthritis. J Rheumatol 2013;40:1349-1356.

53 Helliwell P, Coates L, Chandran V, Gladman D, de Wit M, FitzGerald O, Kavanaugh A, Strand V, Mease PJ, Boehncke W-H, Langley RG, Lubrano E, Maccarone M, Schulze-Koops H, Miceli-Richard C, Queiro R: Qualifying unmet needs and improving standards of care in psoriatic arthritis. Arthritis Care Res 2014;66: 1759-1766.

54 Coates LC, Helliwell PS: Treating to target in psoriatic arthritis: how to implement in clinical practice. Ann Rheum Dis 2016;75: 640-643.

55 Gladman DD, Poulin Y, Adams K, Bourcier M, Barac S, Barber K, Chandran V, Dutz J, Flanagan C, Gooderham MJ, Gulliver WP, Ho VC, Hong C-H, Karsh J, Khraishi MM, Lynde CW, Papp KA, Rahman P, Rohekar S, Rosen CF, Russell AS, Vender RB, Yeung J, Ziouzina O, Zummer M: Treating psoriasis and psoriatic arthritis: position paper on applying the treatto-target concept to Canadian daily practice. J Rheumatol 2017;44:519-534. 
56 Huscher D, Merkesdal S, Thiele K, Zeidler H, Schneider M, Zink A: Cost of illness in rheumatoid arthritis, ankylosing spondylitis, psoriatic arthritis and systemic lupus erythematosus in Germany. Ann Rheum Dis 2006;65: 1175-1183.

57 Bojke L et al.: Modelling the cost-effectiveness of biologic treatments for psoriatic arthritis. Rheumatology (Oxford) 2011;50(suppl 4):iv39-iv47.

58 Poole CD, Lebmeier M, Ara R, Rafia R, Currie CJ: Estimation of health care costs as a function of disease severity in people with psoriatic arthritis in the UK. Rheumatology (Oxford) 2010;49:1949-1956.

59 Brodszky V, Bálint P, Géher P, Hodinka L, Horváth $\mathrm{G}$, Koó É, Péntek M, Polgár A, Seszták M, Szántó S, Ujfalussy I, Gulácsi L: Disease burden of psoriatic arthritis compared to rheumatoid arthritis, Hungarian experiment. Rheumatol Int 2009;30:199-205.

60 Greenberg JD, Palmer JB, Li Y, Herrera V, Tsang Y, Liao M: Healthcare resource use and direct costs in patients with ankylosing spondylitis and psoriatic arthritis in a large US cohort. J Rheumatol 2016;43:88-96.

61 O’Dwyer JL, Meads DM, Hulme CT, Mcparland L, Brown S, Coates LC, Moverley AR, Emery P, Conaghan PG, Helliwell PS: Cost-effectiveness of tight control of inflammation in early psoriatic arthritis: economic analysis of a multicenter randomized controlled trial. Arthritis Care Res 2018;70:462-468.

62 Durham LE, Kirkham BW, Taams LS: Contribution of the IL-17 pathway to psoriasis and psoriatic arthritis. Curr Rheumatol Rep 2015; 17:55.

63 Menon B, Gullick NJ, Walter GJ, Rajasekhar M, Garrood T, Evans HG, Taams LS, Kirkham BW: Interleukin-17+ CD8+ T cells are enriched in the joints of patients with psoriatic arthritis and correlate with disease activity and joint damage progression. Arthritis Rheumatol 2014;66:1272-1281.

64 Res PC et al.: Overrepresentation of IL-17A and IL-22 producing CD8 T cells in lesional skin suggests their involvement in the pathogenesis of psoriasis. PLoS One 2010;5:e14108.

-65 Ortega C, Fernández-A S, Carrillo JM, Romero P, Molina IJ, Moreno JC, Santamaría M: IL17-producing CD8+ T lymphocytes from psoriasis skin plaques are cytotoxic effector cells that secrete Th17-related cytokines. J Leukoc Biol 2009;86:435-443.
66 Villanova F, Di Meglio P, Nestle FO: Biomarkers in psoriasis and psoriatic arthritis. Ann Rheum Dis 2013;72(suppl 2):ii104-ii110.

67 Di Meglio P, Perera GK, Nestle FO: The multitasking organ: recent insights into skin immune function. Immunity 2011;35:857-869. 68 Maeda S et al.: The Th17/IL-23 axis and natu-
ral immunity in psoriatic arthritis. Int J Rheumatol 2012;2012:539683.

69 Chandran V, Cook RJ, Edwin J, Shen H, Pellett FJ, Shanmugarajah S, Rosen CF, Gladman DD: Soluble biomarkers differentiate patients with psoriatic arthritis from those with psoriasis without arthritis. Rheumatology 2010;49: 1399-1405.

70 Dalbeth N, Pool B, Smith T, Callon KE, Lobo M, Taylor WJ, Jones PB, Cornish J, McQueen FM: Circulating mediators of bone remodeling in psoriatic arthritis: implications for disordered osteoclastogenesis and bone erosion. Arthritis Res Ther 2010;12:R164.

-71 Jadon DR, Sengupta R, Nightingale A, Lu H, Dunphy J, Green A, et al.: Serum bone-turnover biomarkers are associated with the occurrence of peripheral and axial arthritis in psoriatic disease: a prospective cross-sectional comparative study. Arthritis Res Ther 2017;19:210.

72 Freeston JE, Coates LC, Nam JL, Moverley AR, Hensor EMA, Wakefield RJ, Emery P, Helliwell PS, Conaghan PG: Is there subclinical synovitis in early psoriatic arthritis? A clinical comparison with gray-scale and power Doppler ultrasound. Arthritis Care Res 2014;66: 432-439.

73 Balint PV et al.: Ultrasonography of entheseal insertions in the lower limb in spondyloarthropathy. Ann Rheum Dis 2002;61:905-910.

74 Gutierrez $\mathrm{M}$ et al.: A sonographic spectrum of psoriatic arthritis: 'the five targets'. Clin Rheumatol 2010;29:133-142.

75 Wiell C, Szkudlarek M, Hasselquist M, Møller JM, Vestergaard A, Nørregaard J, Terslev L, Østergaard M: Ultrasonography, magnetic resonance imaging, radiography, and clinical assessment of inflammatory and destructive changes in fingers and toes of patients with psoriatic arthritis. Arthritis Res Ther 2007; 9:R119.

76 De Simone C et al.: Usefulness of ultrasound imaging in detecting psoriatic arthritis of fingers and toes in patients with psoriasis. Clin Dev Immunol 2011;2011:390726.
77 Sankowski AJ et al.: The comparison of efficacy of different imaging techniques (conventional radiography, ultrasonography, magnetic resonance) in assessment of wrist joints and metacarpophalangeal joints in patients with psoriatic arthritis. Pol J Radiol 2013;78:18-29.

78 Polachek A, Cook R, Chandran V, Gladman DD, Eder L: The association between sonographic enthesitis and radiographic damage in psoriatic arthritis. Arthritis Res Ther 2017;19: 189

79 El Miedany Y et al.: Tailored approach to early psoriatic arthritis patients: clinical and ultrasonographic predictors for structural joint damage. Clin Rheumatol 2015;34:307-313.

80 Mandl P, Navarro-Compán V, Terslev L, Aegerter $\mathrm{P}$, van der Heijde $\mathrm{D}$, D'Agostino MA, Baraliakos X, Pedersen SJ, Jurik AG, Naredo E, Schueller-Weidekamm C, Weber U, Wick MC, Bakker PAC, Filippucci E, Conaghan PG, Rudwaleit M, Schett G, Sieper J, Tarp S, MarzoOrtega H, Østergaard M: EULAR recommendations for the use of imaging in the diagnosis and management of spondyloarthritis in clinical practice. Ann Rheum Dis 2015;74: 1327-1339.

81 Haavardsholm EA et al.: Ultrasound in management of rheumatoid arthritis: ARCTIC randomised controlled strategy trial. BMJ 2016; 354:i4205.

82 Dale J, Stirling A, Zhang R, Purves D, Foley J, Sambrook M, Conaghan PG, van der Heijde D, McConnachie A, McInnes IB, Porter D: Targeting ultrasound remission in early rheumatoid arthritis: the results of the TaSER study, a randomised clinical trial. Ann Rheum Dis 2016;75:1043-1050.

83 Saleem B, Brown AK, Quinn M, Karim Z, Hensor EMA, Conaghan P, Peterfy C, Wakefield RJ, Emery P: Can flare be predicted in DMARD treated RA patients in remission, and is it important? A cohort study. Ann Rheum Dis 2012;71:1316-1321.

84 Brown AK, Conaghan PG, Karim Z, Quinn MA, Ikeda K, Peterfy CG, Hensor E, Wakefield RJ, O’Connor PJ, Emery P: An explanation for the apparent dissociation between clinical remission and continued structural deterioration in rheumatoid arthritis. Arthritis Rheum 2008; 58:2958-2967.

85 Scire CA et al.: Ultrasonographic evaluation of joint involvement in early rheumatoid arthritis in clinical remission: power Doppler signal predicts short-term relapse. Rheumatology (Oxford) 2009;48:1092-1097. 